\title{
Pastoralisme et recours aux marchés : Cas du Sahel sénégalais (Ferlo)
}

\section{Abdrahmane Wane \\ Véronique Ancey \\ Ibra Touré}

\section{Cirad}

Département "Environnements et sociétés " CIRAD-PPZS

Délégation régionale

BP 6189

Dakar-Etoile

99999 Dakar

Sénégal

<awane@cirad.fr>

$<$ veronique.ancey@cirad.fr>

<ibra.toure@cirad.fr>

\section{Résumé}

Souvent perçus comme des êtres contemplatifs non économiques, les pasteurs commercialisent néanmoins leurs produits sur les marchés. Leur principal motif de vente est de dégager des recettes permettant de couvrir leurs dépenses courantes et assurer leur sécurité alimentaire. Au terme d'une enquête sur cinq sites du Ferlo entre la saison des pluies 2005 et celle de 2006, une étude détaillée des ventes des pasteurs montre que la présence de forages, souvent considérée comme élément structurant de l'activité pastorale, n'accroît pas systématiquement les ventes de produits pastoraux. Les recettes sont tirées essentiellement de la commercialisation d'animaux de rente (97,9\% des ventes globales dont bovins à 40\%, petits ruminants à 60\%). Les ventes de produits laitiers (lait frais et caillé, beurre) restent marginales $(0,50 \%)$ et inversement liées au degré d'enclavement des lieux de résidence et d'activité des pasteurs. La diversification observée dans les productions et dans les activités ne se traduit pas dans la commercialisation. Les concepts de pauvreté, de vulnérabilité, de fourniture de services sociaux de base, sont repensés en fonction des caractéristiques du pastoralisme en zones arides (mobilité, éclatement des familles, dispersion de l'habitat).

Mots clefs : commercialisation ; pastoralisme ; pauvreté ; Sahel ; Sénégal.

Thèmes : productions animales ; territoire, foncier ; politiques agricoles et alimentaires.

\section{Abstract \\ Sahelian herders and the markets: Evidence from the Senegalese Sahel (Ferlo)}

Often perceived as non-economic contemplative beings, the pastoral populations of Ferlo appeal to the markets in order to sell their products. Their main goal for selling livestock and animal products consists in making money to cover their usual expenses and to ensure their food safety. Based on primary data obtained through investigations in Ferlo encampments throughout a whole year (winter 2005 - winter 2006), a progressive decomposition of their trade incomes shows that the drillings, often considered by policies as an element which structures the pastoral activity, does not systematically improve the money holdings. The released incomes are related to the marketing of ruminants $(97.9 \%$ of the livestock farming income with the bovines contributing for $40 \%$ and small ruminants for 60\%). The sale of dairy by-products (fresh and curdled milk, butter) remain marginal (0.50\%) and are conversely linked to the degree of enclosure of the herders' places of residence and activity. The diversification of production and the activities is not apparent in the activities of selling. The concepts of poverty, vulnerability and supply of basic social services are reconsidered according to the characteristics of pastoralism in arid regions (mobility, families breakdown, spatial dispersion).

Key words: marketing; pastoralism; poverty; Sahel; Senegal.

Subjects: animal productions; territory, land use; agricultural and food production policy. un des objectifs du Millénaire pour le développement - la réduction de moitié de l'extrême pauvreté avant 2015 - bénéficie d'une attention particulière au Sénégal où le Fond monétaire international (FMI) a 
invoqué en 2002, le manque de revenus et d'accès aux services sociaux de base comme facteurs déterminants. Ce fléau reste préoccupant en milieu rural et les pasteurs du Ferlo sont directement concernés. Ils sont également au centre des interrogations des autorités publiques sur leur capacité à faire face aux prévisions de croissance de la demande globale de viande $(+60 \%)$ et de lait (+ $52 \%$ ) en Afrique d'ici 2020 (Delgado et al., 1999). Dès lors, les autorités sénégalaises affichent la volonté de moderniser l'élevage pastoral en contrôlant les mécanismes de "déstockage ", levier de lutte contre la pauvreté en milieu rural et de satisfaction des besoins nationaux de consommation en produits animaux (ministère de l'Agriculture, de l'Élevage et de l'Hydraulique du Sénégal, 2004). Le terme "déstockage" est toutefois diversement interprété. Pour les services centraux de l'État, il s'agit d'augmenter radicalement la commercialisation du bétail alors que pour les éleveurs, il évoque des images de crises les contraignant à des ventes massives et dramatiques d'animaux à bout de forces, parfois avecl'appui des gouvernements. Les autorités publiques sont confortées dans la voie du " déstockage " par le succès de la politique de promotion de la production locale de viande menée à partir de 2004 . Celle-ci a soutenu le taux de croissance annuel de l'élevage (+ $5 \%$ ) dans un secteur primaire dont les performances annuelles ont décliné de 19,8\% en 2003 à 2,3\% en 2004 mais devrait se relancer pour atteindre $11 \%$ en 2006 (IMF, 2006 ; IMF, 2007).

Cette politique d'élevage est toutefois menée sans connaissance actualisée des comportements de marché des pasteurs. Pour éclairer ces derniers, nous effectuons une analyse économique et spatiale des ventes de produits pastoraux au Ferlo. À l'instar de la plupart des régions pastorales sahéliennes, le Ferlo est déficient en statistiques (Hatfield et Davies, 2006) de sorte qu'un dispositif spécifique et adapté a été imaginé (Wane et al., 2007)

Cet article vise à aborder l'économie pastorale comme un processus dynamique se réalisant au sein et en dehors du marché ; produire des connaissances chiffrées et cartographiées; renseigner la capacité des pasteurs à générer des encaisses monétaires ; montrer l'inadéquation des indicateurs standards de pauvreté dans le contexte pastoral ; contribuer à repenser des services sociaux de base plus adaptés.

Son premier point, méthodologique, décrit le dispositif de collecte des données. Le deuxième expose et spatialise les résultats obtenus. Le troisième discute de leur portée et des perspectives pour le pastoralisme sahélien.

\section{Dispositif de collecte de données adapté} aux populations mobiles

Le Ferlo est un vaste espace d'une superficie de $67610 \mathrm{~km}^{2}$ - soit près d'un tiers du territoire national, délimité par la vallée du fleuve Sénégal au nord et par le front du bassin arachidier au sud. Cet espace désigne parfois abusivement la zone pastorale. En réalité, les pasteurs distinguent les espaces du Fuuta, du Djoloff, du Cayor, du Ferlo, en fonction de multiples critères parmi lesquels la pluviosité n'est qu'un des éléments, sur un transect allant de moins de $300 \mathrm{~mm}$ de pluies annuelles en moyenne à l'Extrême-Nord (zone de départ massif des transhumants) à plus de $400 \mathrm{~mm}$ au sud (zone agropastorale).

Nos enquêtes sur les ventes des pasteurs ont été effectuées entre la saison des pluies de 2005 et celle de 2006 au Ferlo. Elles ont commencé le $1^{\text {er }}$ octobre 2005 (fin de la saison des pluies) et se sont poursuivies jusqu'au 15 juin 2006 en partant des sites du nord du Ferlo vers ceux du sud. L'objectif consistait à obtenir des informations sur les ventes sur une année entière allant de la fin de la saison des pluies de 2004 à celle de 2005.

Aux trois sites (Thiel, Tatki et Rewane) représentatifs de la diversité écologique de cette région (Touré et al., 2003), ont été rajoutés les sites de Boulal, localité proche de Dahra (gros marché régional de regroupement et d'échange de bétail), et de Mbame assez enclavé pour renseigner sur l'activité pastorale en l'absence de toute marque de politique publique. Tous les sites disposent de forages pastoraux (représentés dans les figures ci-après par le centre du premier cercle intérieur) excepté Mbame où il n'y a que des puits pastoraux privatifs.

Les enquêtes ont été menées dans des campements au sein desquels les principaux répondants étaient les chefs de ménages et leurs épouses. La distinction entre campements et ménages découle du fait que la famille pastorale est constituée d'un ensemble de ménages (en peul : kiralé au pluriel, hirandé au singulier) vivant dans des campements (galle, galleji) qui sont des unités de gestion et de production.

Après traitement des données d'enquête, l'étude porte finalement sur un échantil-

\section{Tableau 1. Distribution de l'échantillon entre les sites.}

Table 1. Sample distribution between sites.

\begin{tabular}{lccccc}
\hline Sites focaux & $\begin{array}{c}\text { Répartition initiale } \\
\text { des campements }\end{array}$ & $\begin{array}{c}\text { En proportion } \\
\text { (\%) }\end{array}$ & $\begin{array}{c}\text { Taille théorique } \\
\text { d'échantillon }\end{array}$ & $\begin{array}{c}\text { Taille effective } \\
\text { d'échantillon }\end{array}$ \\
\hline Marge d'erreur ${ }^{1}$ & & & $3 \%$ & $5 \%$ & $4,68 \%$ \\
Mbame & 83 & 11 & 49 & 28 & 23 \\
Boulal & 268 & 36 & 158 & 91 & 79 \\
Rewane & 121 & 16 & 71 & 40 & 54 \\
Tatki & 105 & 14 & 62 & 36 & 64 \\
Thiel & 163 & 22 & 96 & 56 & 56 \\
Total & $\mathbf{7 4 0}$ & $\mathbf{1 0 0}$ & $\mathbf{4 3 8}$ & $\mathbf{2 5 3}$ & $\mathbf{2 7 6}$ \\
\hline
\end{tabular}

${ }^{1}$ Pour un indice de confiance de $95 \%$ et une proportion de $50 \%$. 
lon de 276 campements (tableau 1) dont $20 \%$ se trouvent sur le site de Rewane, $29 \%$ à Boulal, $20 \%$ à Thiel, $23 \%$ à Tatki et $8 \%$ à Mbame. Cet échantillon correspond à une marge d'erreur de 4,68\%, un intervalle de confiance de $95 \%$, une proportion de $50 \%$. Il répond aux standards statistiques en matière d'échantillonnage (Anderson et al., 2001)

\section{Structure des ventes des pasteurs}

L'examen de la structure des ventes des pasteurs s'effectuera à trois niveaux : le global, la moyenne par site et la moyenne par campement.

\section{Analyse de la répartition des recettes globales}

D'après les déclarations des chefs de ménage, les ventes réalisées entre la fin de la saison des pluies 2004 et celle de 2005 représentent 692 millions de F CFA (1,055 million d'euros). Ces recettes proviennent des ventes d'animaux de rente (bovins, ovins, caprins) à 97,9\%; des produits laitiers (lait frais, lait caillé et beurre) à $0,5 \%$; des asins et équins à $0,8 \%$ et des cultures (mil, gomme arabique, produits de cueillette - jujubes, fruits de balanites) à $0,8 \%$ (tableau 2).

Les recettes de vente des animaux de rente s'élèvent environ à 677 millions de F CFA (1,032 million d'euros) avec une contribution des bovins à $40 \%$, des ovins à $53 \%$ et des caprins à $7 \%$ (tableau 2).
Les ventes de produits laitiers contribuent marginalement (un peu moins de 4 millions de F CFA soit 6098 euros) aux recettes globales des pasteurs. Les produits laitiers vendus sont constitués de denrées rapidement périssables (lait frais et caillé) ou relativement stockables sur une année (beurre). Les ventes portent sur le lait frais (19\%), le lait caillé (31\%) et le beurre (50\%) (tableau 2). La consommation des produits laitiers et leur vente jouent un rôle vital dans la sécurité alimentaire des ménages pastoraux. Leur commercialisation reste, cependant, fortement contrainte par le déficit d'infrastructures de collecte et de stockage, le caractère saisonnier de ces produits, le niveau élevé des coûts de transactions et également les comportements des pasteurs inscrits dans une logique de sécurisation de leur consommation. Il ressort des entretiens qu'un arbitrage permanent s'effectue entre autoconsommation et commercialisation.

\section{Répartition des recettes commerciales par site}

Près de $57 \%$ des recettes globales proviennent de Tatki et de Boulal qui fournissent $52 \%$ des campements enquêtés. Avec seulement $8 \%$ des campements, Mbame contribue à hauteur de $12 \%$ aux recettes globales alors qu'avec chacun $20 \%$ des campements, Thiel et Rewane ne participent qu'à hauteur de 18 et $13 \%$. La spatialisation des résultats montre une très forte hétérogénéité des recettes sur les sites disposant de forages (Tatki, Rewane et Boulal) mais une certaine homogénéité sur le seul site doté de puits pastoraux (Mbame). En revanche, les ventes élevées (de 4,2 à 18,5 millions de F CFA soit 6402 à 28203 d'euros) sont concentrées à Mbame (39\%) et Tatki (23\%) alors que les faibles recettes (moins de 2 millions de F CFA soit 3049 euros) se retrouvent en majorité autour des forages pastoraux à Rewane (76\%), Boulal (63\%) et Thiel (61\%) (figure 1).

La commercialisation des animaux de rente procure plus de $96 \%$ des ventes globales par site. Les ventes d'asins et d'équins sont plus fortes à Mbame où, avec l'éparpillement de la population et l'absence d'infrastructures de base, ces animaux sont utilisés pour les déplacements des pasteurs vers les marchés et les points d'eau.

Sur l'ensemble des sites enquêtés, la commercialisation des cultures contribue marginalement aux recettes. Cette situation est aussi valable pour les sites de Thiel et de Rewane qui sont pourtant situés dans des zones agropastorales. Les ventes des cultures y contribuent à plus de $2 \%$ des recettes globales (tableau 2). À Thiel, il s'agit de vente de mil et d'arachide alors qu'à Rewane, zone de forte déprise agricole, nous avons plutôt observé lors de nos enquêtes, des produits de cueillette et de la gomme arabique même si les déclarations de ventes sont probablement sous-estimées car ces activités sont plutôt mal considérées socialement. Comme pour le lait, un arbitrage entre autoconsommation et commercialisation est effectué sur les produits agricoles par les agropasteurs qui cultivent de plus en plus dans la zone sud du Ferlo. L'activité agricole s'organise sur un " clivage " entre une production animale relativement destinée au marché (avec les limites imposées par les contraintes de sécurité alimentaire en milieu pastoral) et des cultures vivrières destinées à l'autoconsommation. Les ventes de cultures sont quasi inexistantes à Mbame.

\section{Tableau 2. Détail des ventes de produits pastoraux.}

Table 2. Breakdown of pastoral product sales.

\begin{tabular}{|c|c|c|c|c|c|c|c|c|c|c|c|}
\hline Ventes & $\begin{array}{c}\text { Total } \\
\text { (millions } \\
\text { F CFA) }\end{array}$ & $\begin{array}{c}\text { Animaux } \\
\text { de rente (\%) }\end{array}$ & $\begin{array}{c}\text { Bovin } \\
(\%)\end{array}$ & $\begin{array}{c}\text { Ovins } \\
\text { (\%) }\end{array}$ & $\begin{array}{c}\text { Caprins } \\
\text { (\%) }\end{array}$ & $\begin{array}{l}\text { Équidés } \\
(\%)\end{array}$ & $\begin{array}{c}\text { Produits } \\
\text { laitiers } \\
(\%)\end{array}$ & $\begin{array}{l}\text { Lait frais } \\
\text { (\%) }\end{array}$ & $\begin{array}{l}\text { Lait } \\
\text { caillé } \\
(\%)\end{array}$ & $\begin{array}{c}\text { Beurre } \\
(\%)\end{array}$ & $\begin{array}{c}\text { Cultures } \\
(\%)\end{array}$ \\
\hline Boulal & 175,2 & 97,6 & 53,8 & 40,3 & 4,8 & 1,1 & 1,1 & 36,6 & 43,0 & 20,4 & 0,2 \\
\hline Mbame & 84,9 & 98,3 & 32,0 & 60,1 & 6,3 & 1,7 & 0,0 & 0,0 & 0,0 & 100,0 & 0,0 \\
\hline Rewane & 90,8 & 97,5 & 35,9 & 50,7 & 13,1 & 0,3 & 0,2 & 0,0 & 6,1 & 93,9 & 2,0 \\
\hline Tatki & 215,9 & 99,2 & 33,8 & 60,0 & 5,8 & 0,4 & 0,4 & 0,2 & 11,1 & 88,7 & 0,1 \\
\hline Thiel & 125,2 & 96,1 & 36,7 & 54,1 & 8,3 & 0,9 & 0,6 & 0,0 & 26,4 & 73,6 & 2,4 \\
\hline Total & 692,0 & 97,9 & 39,4 & 52,8 & 7,0 & 0,8 & 0,5 & 19,0 & 31,0 & 50,0 & 0,8 \\
\hline
\end{tabular}

1 euro $=655,96$ F CFA. 


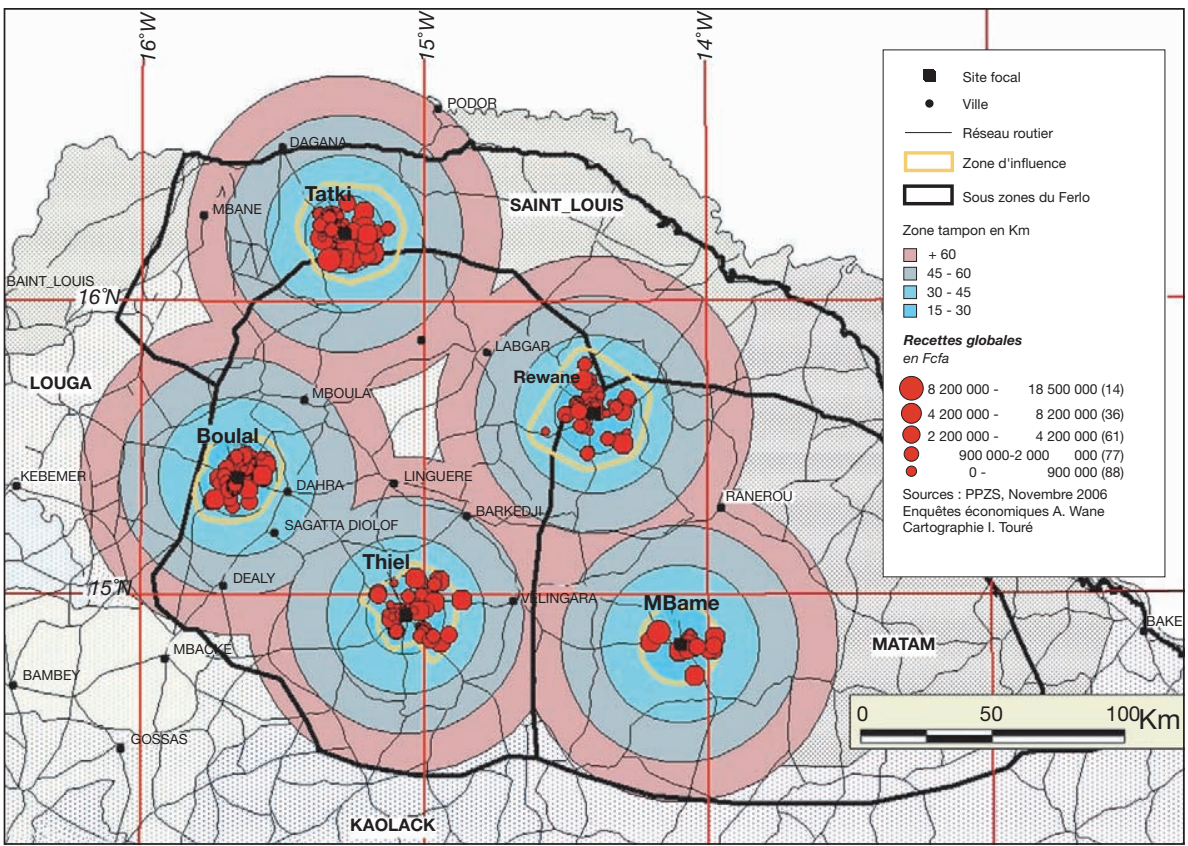

Figure 1. Zone d'enquête : ventes globales par site.

Figure 1. Study zone: per site global sales.

En termes de structures de vente par espèce animale, les ventes de bovins concernent essentiellement des bœufs (58-75\% selon les sites). Les vaches sont relativement moins commercialisées du fait de la variété de bénéfices qu'elles procurent aux pasteurs (lait et veaux). La surreprésentation des bœufs dans les ventes animales est plus marquée à Rewane (3/4 mâles pour 1/4 de femelles) qu'à Boulal. Les ventes d'ovins concernent davantage les moutons (67-80\%) que les brebis (33$20 \%$ ). La prédominance des ventes d'ovins mâles s'explique par les pics observés lors des fêtes de l'Aïd-El-Kébir, suivant la recommandation de l'Islam de sacrifier des moutons, et aussi par la préférence générale à garder une majorité de reproductrices. Pour les caprins, il y a plus de chèvres vendues que de boucs, sauf à Boulal. (figure 2). L'effet prix est favorable à la vente de chèvres, meilleur marché, souvent écoulées dans les "dibiteries " (restaurants populaires spécialisés dans la vente de viande grillée).

Grâce à la proximité de la route goudronnée, la commercialisation des produits laitiers est plus importante à Boulal. Elle est marquée à $80 \%$ par les ventes de lait frais et caillé, à l'inverse des sites plus ou moins enclavés. Il y a donc une diversification des ventes dans les sites disposant d'infrastructures de base et à proximité des marchés et des pistes. La différence entre Boulal et Thiel s'explique également par la proximité du marché de Dahra. Le plus gros marché régional de regroupement de bétail joue un rôle spécifique sur les ventes comparativement au rôle limité des marchés de brousse (luma) tels que Thiel. Dans les sites isolés comme Mbame, les ventes concernent exclusivement les produits stockables (beurre) (figure 3).

\section{Analyse des ventes par campement}

Les recettes annuelles s'élèvent en moyenne par campement à 2507386 F CFA (3 822 euros) et par tête à 147462 F CFA (225 euros). Comme les recettes par campement intègrent l'ensemble des individus, une première approximation de la productivité des actifs est obtenue par les recettes moyennes par actif déclaré travailler dans l'élevage (284 $321 \mathrm{~F}$ CFA ou 433 euros).

Quelques grandes tendances sont observables au niveau des campements. À Rewane (figure 4) et à Tatki, les campements à recettes élevées tendent à s'implanter loin des forages pour éviter la concurrence sur les pâturages. Ceux à faibles recettes se situent souvent à proximité des pistes et cette tendance se renforce avec la proximité des forages. En revanche, les deux tendances précéden- tes sont diluées à Boulal (figure 5). La configuration spatiale des campements subit la double influence du forage et de la route bitumée Louga-Dahra. Certains gros éleveurs s'installent de part et d'autre de la route nationale et du forage pour écouler plus facilement leurs produits notamment laitiers.

\section{Discussion}

\section{et perspectives pour l'économie du pastoralisme}

Notre analyse des ventes de produits animaux au Ferlo inscrit le pastoralisme dans un champ d'investigation intégrant la singularité des comportements de marché des pasteurs. Elle révèle le niveau de sensibilité des pasteurs aux opportunités des marchés. Elle étaye quantitativement une forme de rationalité contingente à un environnement incertain. Les pasteurs s'adressent généralement aux marchés pour y réaliser une recette prédéterminée permettant de couvrir leurs dépenses de consommation courante. Ils sont tout aussi capables de saisir des opportunités lorsque les signaux de marchés leur apparaissent favorables (Aïd-El-Kébir). L'imperfection et l'incomplétude des informations de marchés les incitent à une position prudente, adaptée aux circonstances et donc, contingente à leur environnement socio-économique (Wane, 2005). Cela explique que les pasteurs ne sont pas dans une logique de "déstockage " régulier de leurs animaux même si les services techniques les y incitent. Loin de se désintéresser du niveau des prix du marché (Kerven, 1992), les éleveurs opèrent des arbitrages entre leurs nécessités immédiates et leurs besoins anticipés de moyen terme. Par analogie avec les différentes formes de capital, les divers types de bétail peuvent être assimilés, selon les situations, à de la trésorerie, à une assurance et à un patrimoine, d'où une commercialisation "mesurée" en nombre d'unités vendues comme en décisions temporelles de mises sur le marché. Ce qui importe pour eux c'est de s'insérer dans le marché tout en conservant du cheptel en réserve afin de parer à certains risques. C'est en période de crise et à 


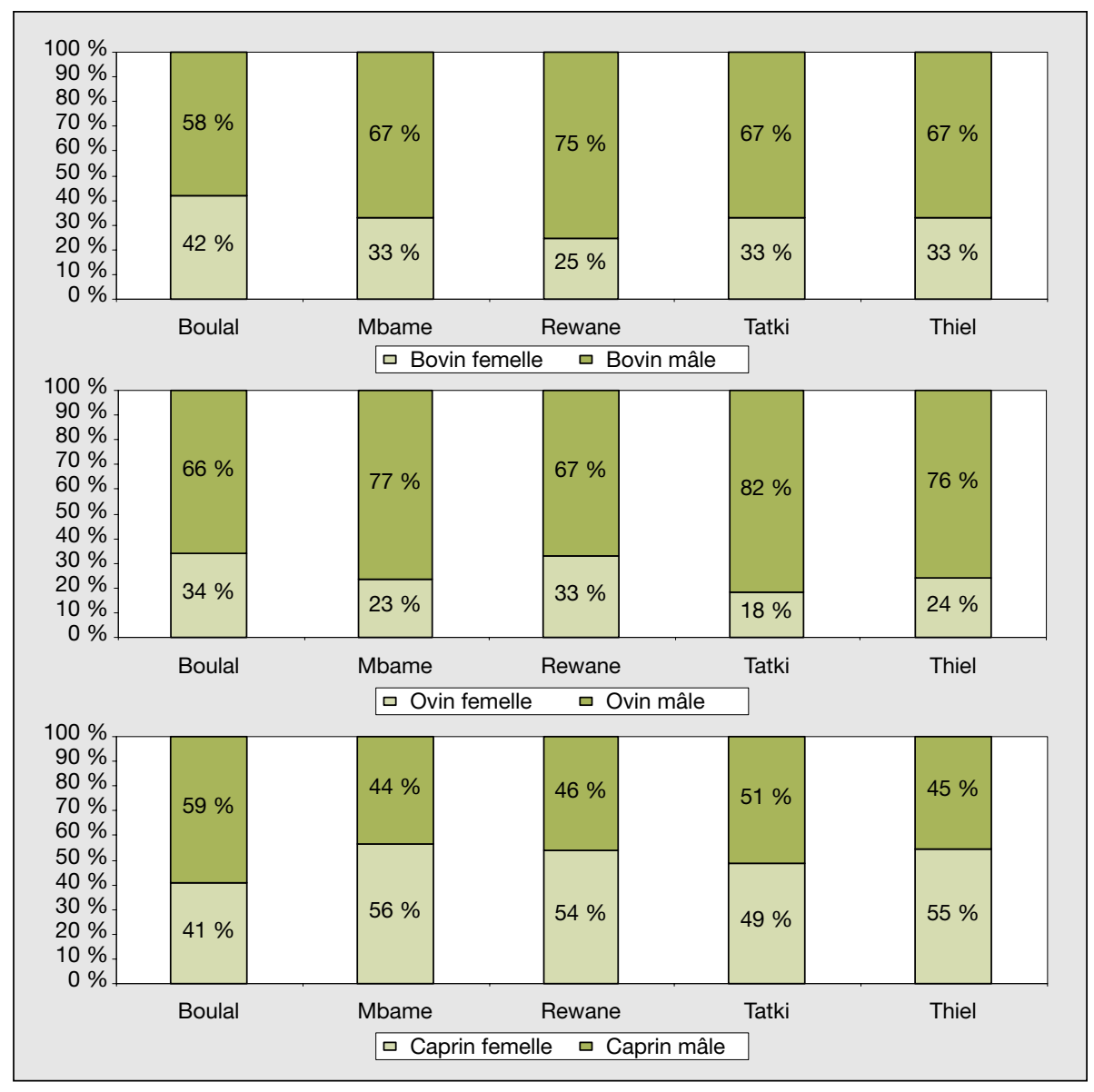

Figure 2. Répartition des ventes d'espèces animales par site.

Figure 2. Distribution of the animal species sales per site.

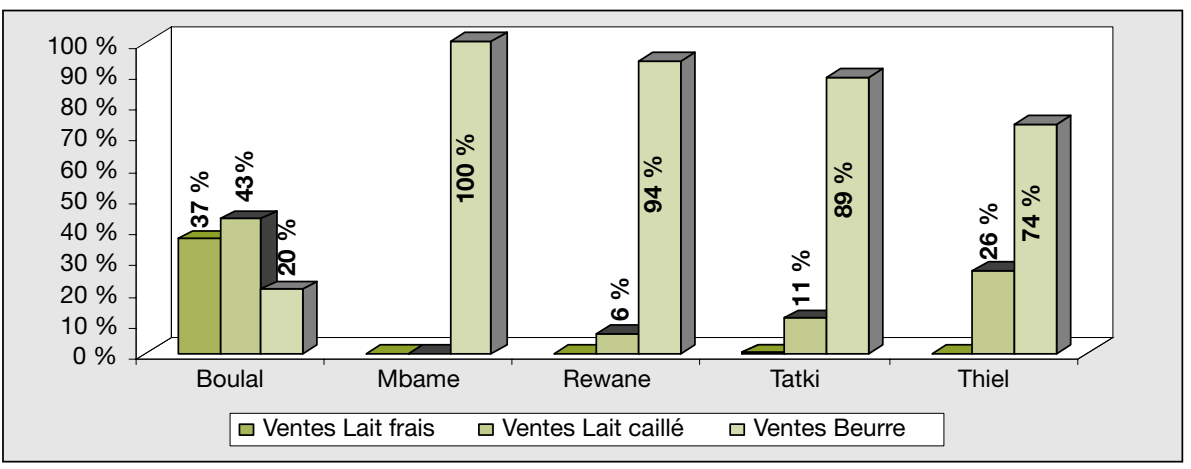

Figure 3. Répartition des ventes de produits laitiers par site.

Figure 3. Distribution of dairy product sales per site.

défaut de pouvoir nourrir le bétail et/ou à cause de la flambée des prix des céréales que les éleveurs se sentent contraints de " déstocker ", vivant à un degré élevé une tension classique entre les nécessités du marché et celles d'une société précapitaliste.
Cette situation conduit à un conflit d'intérêts entre des autorités sénégalaises soucieuses d'approvisionner les grands centres urbains en viande et en lait et des pasteurs confrontés d'abord à leur sécurité alimentaire (Ancey et Monas, 2005). De plus, les décideurs politiques se préoccupent des importations massives de lait en poudre (152 159 tonnes en moyenne entre 2000 et 2005) face à une demande potentielle de 267598 tonnes (Wane, 2006) et aussi de la faiblesse de la consommation de lait par habitant qui, en 2003, était de 27 litres par an (Corniaux, 2003).

Les utilités du bétail, leur valeur et leurs modes de circulation dépassent la seule fonction marchande en société pastorale. Dans ce contexte, parler de pauvreté monétaire (IMF, 2002) paraît inapproprié. Compte tenu de la commercialisation orientée et autolimitée aux besoins courants des pasteurs et de leur famille et des nombreuses ressources non monétaires dont dépend leur sécurité, la notion de vulnérabilité est plus pertinente et rend mieux compte, à la fois, de la diversité des besoins, de l'exposition aux risques, et de la dynamique des stratégies des acteurs déterminant leur réactivité.

Comme autre déterminant important de la pauvreté au Sénégal, le FMI a identifié l'accès aux services sociaux de base des populations rurales. En milieu pastoral notamment, les niveaux de scolarisation sont faibles et les services de santé se dégradent. Cela est dû, en partie, au dysfonctionnement de ces services mais renvoie également aux difficultés d'accès particulières des ruraux dispersés et mobiles. Les services sociaux à réhabiliter devraient intégrer les caractéristiques du pastoralisme sahélien. Une fourniture de services sociaux de base ajustée aux réalités pastorales passerait par une politique foncière équilibrée entre les intérêts des usagers, une scolarisation adaptée (cantines scolaires, hébergement, véhicules scolaires mobiles en saison de transhumance), des innovations financières (micro-crédit et micro-assurance).

L'analyse des comportements de marché des pasteurs permet enfin de contribuer aux débats inspirés par Polanyi (1983) sur l'imbrication de la production et de l'échange dans le politique, le social et le culturel. Dans la résistance des pasteurs au "déstockage" se joue la reproduction et la durabilité socio-économique de leur système d'élevage, tant que le troupeau hérité, géré et valorisé au sein et hors du marché représente le principal pilier de sécurité alimentaire, d'assurance et de reproduction sociale, dans une société par ailleurs sensible aux opportunités. La modernisation des sociétés et économies pastorales, si souvent invoquée, n'est pas strictement une affaire d'augmentation de la production marchande 


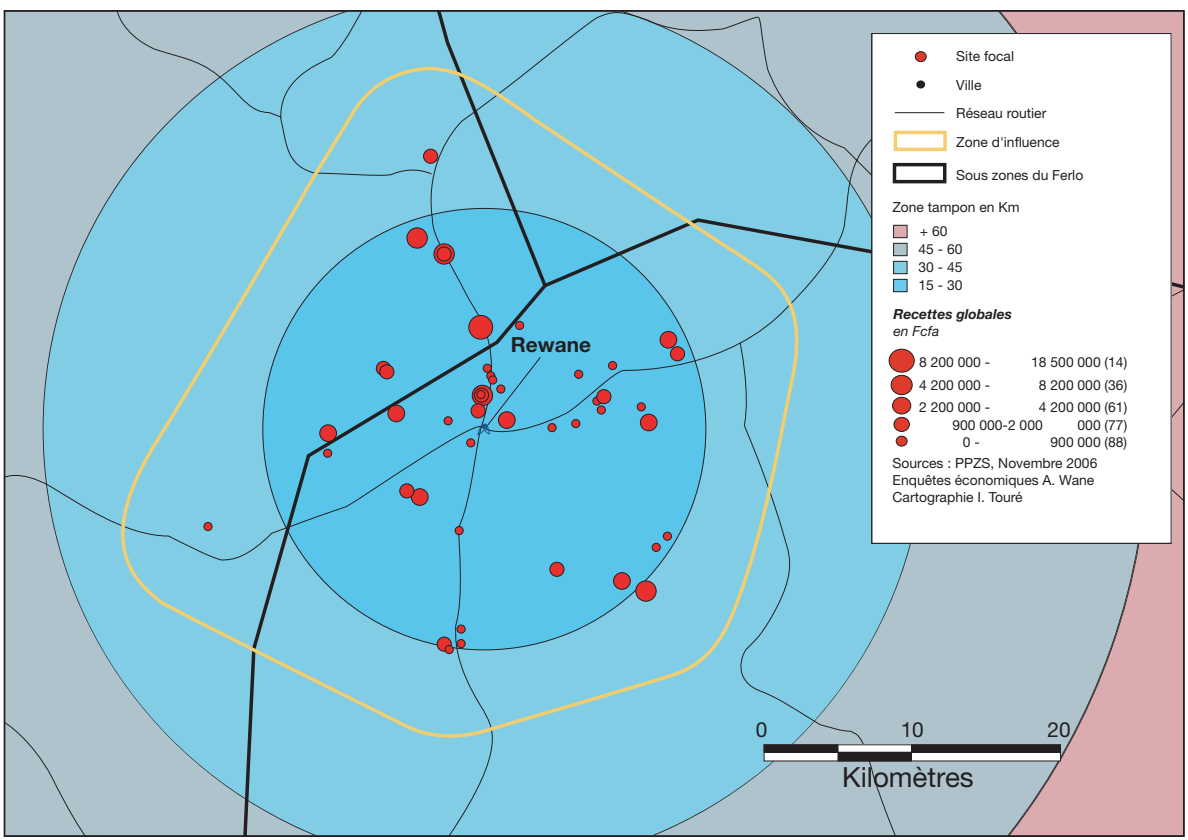

Figure 4. Rewane. Ventes globales par campement.

Figure 4. Rewane: per encampment global sales.

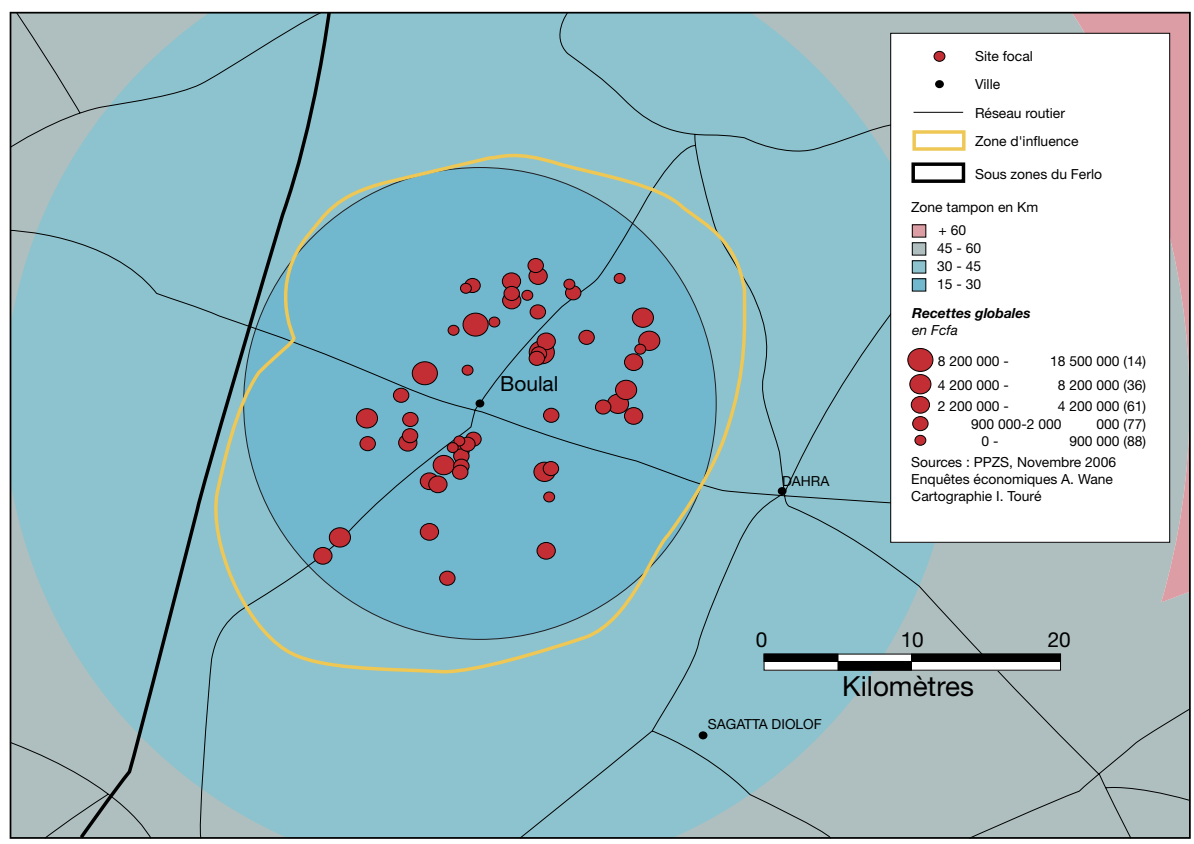

Figure 5. Boulal : ventes globales par campement.

Figure 5. Boulal: per encampment global sales.

mais avant tout d'augmentation de la sécurité et du bien-être des populations, sans laquelle les ambitions légitimes des politiques sectorielles ne se réaliseront pas durablement. les éleveurs à favoriser la commercialisation de leurs produits. La diversification de la production pastorale (produits animaux, dérivés et agricoles) ne se traduit pas totalement sur le plan commercial. Cela détermine des équilibres économiques et alimentaires différents dans la région. De plus, les différents profils statistiques de la structuration des revenus dans les campements suggèrent que les équilibres sociaux dans les unités pastorales se diversifient eux aussi en fonction des responsabilités individuelles de production et de gestion des revenus.

Le recours autolimité aux marchés révèle un comportement des pasteurs qui, dans un environnement incertain, savent profiter des opportunités de marché sans chercher à "déstocker". Le terme de "pauvreté monétaire" est moins adapté que celui de vulnérabilité compte tenu des besoins monétaires et des stratégies de marché des pasteurs.

Notre analyse statistique, économique et spatiale des ventes pastorales renforce la connaissance actuelle de l'économie pastorale des zones arides du Sénégal. Elle montre que cette économie se réalise à la fois au sein et hors des marchés. Elle attire aussi l'attention sur la gestion des ventes comme un facteur stratégique en complément des enjeux de la gestion des ressources naturelles.

\section{Références}

Ancey V, Monas G. Le pastoralisme au Sénégal entre politique " moderne " et gestion des risques par les pasteurs. Revue Tiers Monde. $X$ LVI 2005 ; 184 : 761-83.

Anderson DR, Sweeney DJ, Williams TA. Statistiques pour l'économie et la gestion, Trad.

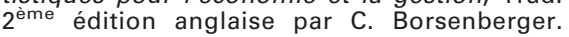
Coll. Ouvertures Economiques. Paris: De Boeck University s.a., 2001

Corniaux C. La filière lait et produits laitiers dans la région de Saint-Louis. Saint-Louis (Sénégal) : Cirad-EMVT; Isra, 2003. www.infoconseil.sn/IMG/laitStLouis.pdf

Delgado C, Rosegrant M, Steinfeld H, Ehui S, Courbois C. Livestock to 2020: The Next Food Revolution 2020. Washington, DC : International Food Policy Research Institute (IFPRI), 1999.

IMF. Republic of Senegal poverty reduction strategy paper. Washington : IMF, 2002. www. imf.org/external/np/prsp/2002/sen/01/

IMF. Senegal: Poverty reduction strategy paper - Second annual progress report. IMF Country Report 2006: 06/68. Washington : IMF, 2006. http://planipolis.iiep.unesco.org/upload/Senegal/PRSP/Senegal\%20PRSP\%20Second\% 20Annual\%20Progress\%20Report\%202005.pd 
IMF. Senegal: Poverty reduction strategy paper. IMF Country Report 2007; 07/316. Washington IMF, 2007. http://planipolis.iiep.unesco.org/ upload/Senegal/Senegal_PRSP_2006.pdf

Hatfield R, Davies J. Global review of the economics of pastoralism. Report Prepared for the WISP (World Initiative for Sustainable Pastoralism) with contributions from Wane A, Kerven C, Dutilly-Diane C, Biber JP, Merega JL, Odhiambo MO, Behnke R, Gura S. Nairobi IUCN, 2006. http://cmsdata.iucn.org/downloads/global_review_ofthe_economicsof_pastoralism_fr.pdf

Kerven C. Customary commerce: a historical reassessment of pastoral livestock marketing in Africa. London : Overseas Development Institute, 1992.
Touré I, Diop AT, Diouf A. Dynamic analysis of landscapes and landcovers for the knowledge and evolution of the pastoral ecosystems in the Ferlo-Senegal. In : Proceeding of the VII International Rangelands Congress. Durban: Editions N. Allsopp, 2003

Wane A, Touré I, Toutain B, Ancey V, Diop AT, Ickowicz $A$. How to obtain a representative sample of economic studies in the areas with strong mobility? Case of the Senegalese Sahel (Ferlo). In : Donatelli M, Hatfield J, Rizzoli A, eds. Farming Systems Design. Book 2 - Fieldfarm scale design and improvement. 2007 International Symposium on Methodologies on Integrated Analysis on Farm Production Systems, Catania (Italy), 10-12 September 2007.
Wane A. Review on the literature on pastoral economics and marketing: West Africa. Prepared for the WISP (World Initiative for Sustainable Pastoralism). Nairobi : IUCN, 2006. http://cmsdata.iucn.org/downloads/west africa_reports.pdf

Wane A. Marchés de bétail du Ferlo (Sahel sénégalais) et comportements des ménages pastoraux. Actes Colloque SFER, Montpellier, 7-9 novembre 2005. 